\title{
IMPACT OF HEURISTIC STRATEGIES ON PUPILS' ATTITUDES TO PROBLEM SOLVING
}

Jarmila Novotná ${ }^{1}$ Petr Eisenmann ${ }^{2}$, Jiří Přibyl ${ }^{2}$

${ }^{1}$ Charles University in Prague, ${ }^{2}$ University of J. E. Purkyně Ústí nad Labem

Highlights
- The strategy Introduction of an auxiliary element can be mastered in a shorter period of time
- The strategy Omitting a condition requires longer teacher's work with pupils
- A short period of using heuristic strategies changes pupils' attitudes to problem solving

\section{Abstract}

The paper is a sequel to the article (Novotná et al., 2014), where the authors present the results of a 4-month experiment whose main aim was to change pupils' culture of problem solving by using heuristic strategies suitable for problem solving in mathematics education. (Novotná et al., 2014) focused on strategies Analogy, Guess - check - revise, Systematic experimentation, Problem reformulation, Solution drawing, Working backwards and Use of graphs of functions. This paper focuses on two other heuristic strategies convenient for improvement of pupils' culture of problem solving: Introduction of an auxiliary element and Omitting a condition. In the first part, the strategies Guess - Check - Revise, Working backwards, Introduction of an auxiliary element and Omitting a condition are characterized in detail and illustrated by examples of their use in order to capture their characteristics. In the second part we focus on the newly introduced strategies and analyse work with them in lessons using the tools from (Novotná et al., 2014). The analysis of results of the experiment indicates that, unlike in the case of the strategy Introduction of an auxiliary element, successful use of the strategy Omitting a condition requires longer teacher's work with the pupils. The following analysis works with the strategy Systematic experimentation, which seemed to be the easiest to master in (Novotná et al., 2014); we focus on the dangers it bears when it is used by pupils. The conclusion from (Novotná et al., 2014), which showed that if pupils are introduced to an environment that supports their creativity, their attitude towards problem solving changes in a positive way already after the period of four months, is confirmed.

\section{Keywords}

Problem Solving, Solving Strategies, Guess - check - revise, Systematic experimentation, Introduction of an auxiliary element, Omitting a condition

Novotná J., Esenmann P. and Přibyl J. (2015) “Impact of Heuristic Strategies on Pupils’ Attitude to Problem Solving”, Journal on Efficiency and Responsibility in Education and Science, Vol. 8, No. 1, pp. 15-23, online ISSN 1803-1617, printed ISSN 2336-2375, doi: 10.7160/eriesj.2015.080103.

\section{Introduction}

It is a fact generally agreed on by teachers, mathematics educators and researchers in the field of mathematics education that problem solving is the cornerstone of mathematics taught at all school levels. The posed problems should be more than tasks in which an individual or a group are meant to demonstrate to their teacher how they can apply mastered algorithms. Problems should develop pupils' intellectual activity, simulate work of a mathematician who is facing a problem to be solved, encourage solvers' creativity in the solving process (Brousseau, Novotná, 2008). If problems are to meet these criteria, it is not enough to look for good assignments (although we cannot do without them). It is essential to create suitable environments that influence pupils' relationship to problem solving. We refer to this environment as stimulating learning environment in contrast to Wittmann's (1995) concept substantial learning environment which refers to the context from which mathematics problems arise naturally.

Changes of pupils' attitude to problem solving are one of the phenomena studies in the frame of the GAČR research project Development of culture of problem solving in mathematics in Czech schools. The project explores the possible ways of changing pupils' attitudes to problem solving, of making pupils aware that mathematics problems are the means needed for their own personal development. One of the main questions the research team is trying to answer is the question to which extent this attitude positively influences development of pupils' understanding of mathematics, their approach to creative search for solutions to problems, and their behaviour once they encounter a modified or brand new problem.

\section{Theoretical background}

In (Novotná et al, 2013; Břehovský et al, 2013; Novotná et al, 2014), the focus is especially on introduction of heuristic strategies suitable for use in primary and secondary schools when solving problems differently than with school algorithms. The presence of non-routine algorithms in mathematics classrooms in China, Singapore and USA is researched in (Fan \& Zhu, 2007). (Elia, van den Heuvel-Panhuizen \& Kolovou, 2009) describe an experiment exploring improvement of the ability to solve problems after teaching heuristic strategies by primary school high achievers.

However, most pupils cannot be expected to start using these heuristic strategies unless they are given help, albeit from the teacher or somebody else from outside the school. There are many ways to create of learning environment to make it more stimulating. One of them is the inclusion of problems whose solution is much easier or more convenient if pupils use some 
(or one) heuristic strategies instead of algorithmic solution typically used in school mathematics.

Creation of a stimulating learning environment in mathematics is not and cannot be a single event. The question is how long a teacher must exert their influence on pupils until it brings positive changes in their attitude to solving of mathematics problems, improves their understanding of mathematics and until their ability to use mathematics in different situations is developed. The paper presents one of the possible approaches to this issue. It reports on a four month teaching experiment described already in (Novotná et al., 2014), in which pupils were repeatedly introduced to the advantages of use of heuristic strategies Analogy, Guess - check - revise, Systematic experimentation, Problem reformulation, Solution drawing, Working backwards and Use of graphs of functions in solving mathematics problems and in which changes in their attitudes to problem solving were detected. It was proved that if pupils are introduced to an environment that supports their creativity, their attitude towards problem solving changes in a positive way already after the period of four months. The most successful of all the studied heuristic strategies used by the pupils were the strategies Guess - check - revise and Systematic experimentation.

The focus of this article is on two other heuristic strategies that can be used on secondary levels: Introduction of an auxiliary element and Omitting a condition. Their use and effect on improvement of pupils' culture of problem solving are subjected to the same analyses as strategies in (Novotná et al., 2014). Attention is also paid to the strategy Systematic experimentation, which was the strategy most frequently used by pupils in the experiment, and the dangers its use represents to pupils.

(Novotná et al., 2014) describes the strategies Analogy, Guess - check - revise, Systematic experimentation, Problem reformulation, Solution drawing, Working backwards and Use of graphs of functions only briefly. In this paper, the types of problems that can be solved efficiently using these strategies and the potential dangers a pupil must face when using the strategies are illustrated on real problems. A detailed description of the five strategies that were used in the four-month-experiment is presented below. This will allow the reader to grasp the core of the different strategies.

\section{Materials and Methods}

11 teaching experiments were conducted in 11 lower and upper secondary schools in North Bohemia in the years 2012 and 2013. 4 experiments were conducted with 12-year-old pupils, 4 experiments with 14-year-old pupils and 3 experiments with 17-year-old students. All the selected schools were ordinary schools without any specialization (basic schools in case of 12 and 14 year old pupils and upper secondary grammar schools in case of 17 year old pupils). The classes were characterized as average or even slightly below average by their teachers.

In the period of four months, teachers of mathematics tried to teach their pupils to use two or three of the bellow listed heuristic strategies when solving mathematics problems.

\section{Systematic experimentation}

The principle behind this strategy is the idea that the result may be reached in a finite number of attempts. These attempts are carried out systematically. Each subsequent attempt will be "slightly" modified with respect to the previous one. The principle is based on the fact that the solver selects an initial value and then gradually gets nearer to the sought solution. This strategy seems to be very efficient in connection with the use of technological devices as these make it possible to conduct the experiments in real time. The use of computer in systematic experimentation is sometimes referred to as the use of "brute" force. The solution by brute force is specified as exhausting all single possibilities from the set of all potential results. The specification allows us to classify the presented illustrative problems into the following categories:

1. Problems solved using brute force (exploring all the possibilities).

2. Problems solved using brute force with justified termination of the experiment (exploring only a selected subset of all possible solutions).

A specific group is formed by problems in which

3. Systematic experimentation results in formulation or verification of some hypothesis.

Let us now illustrate the described strategy by one solution of a problem from each of the three above listed categories.

\section{Problem 1}

Assignment: Some tickets in a theatre were sold for $220 \mathrm{CZK}$ and some at the price of $160 \mathrm{CZK}$. How many tickets at each price were sold if the total sum of 97 tickets was $19300 \mathrm{CZK}$ ?

Solution: A spreadsheet will be used for experimenting. As mentioned above there are two possibilities. The first one starts as follows (see Table 1):

\begin{tabular}{|c|c|c|c|c|}
\hline $\begin{array}{c}\text { Number of tickets } \\
\text { at } 220 \mathrm{CZK}\end{array}$ & $\begin{array}{c}\text { Price in } \\
\mathrm{CZK}\end{array}$ & $\begin{array}{c}\text { Number of tickets } \\
\text { at } 160 \mathrm{CZK}\end{array}$ & $\begin{array}{c}\text { Price in } \\
\mathrm{CZK}\end{array}$ & $\begin{array}{c}\text { Total price } \\
\text { in CZK }\end{array}$ \\
\hline 97 & 21340 & 0 & 0 & 21340 \\
\hline
\end{tabular}

Table 1: Starting the first possibility for experimenting and the other starts by a well-founded estimation (see Table 2).

\begin{tabular}{|c|c|c|c|c|}
\hline $\begin{array}{c}\text { Number of tickets } \\
\text { at 220 CZK }\end{array}$ & $\begin{array}{c}\text { Price in } \\
\text { CZK }\end{array}$ & $\begin{array}{c}\text { Number of tickets } \\
\text { at 160 CZK }\end{array}$ & $\begin{array}{c}\text { Price in } \\
\text { CZK }\end{array}$ & $\begin{array}{c}\text { Total price } \\
\text { in CZK }\end{array}$ \\
\hline 65 & 14300 & 32 & 5120 & 19420 \\
\hline 64 & 14080 & 33 & 5280 & 19360 \\
\hline $\mathbf{6 3}$ & $\mathbf{1 3 8 6 0}$ & $\mathbf{3 4}$ & $\mathbf{5 4 4 0}$ & $\mathbf{1 9 3 0 0}$ \\
\hline 62 & 13640 & 35 & 5600 & 19240 \\
\hline$\ldots$ & $\ldots$ & $\ldots$ & $\ldots$ & $\ldots$ \\
\hline 1 & 220 & 96 & 15360 & 15580 \\
\hline 0 & 0 & 97 & 15520 & 15520 \\
\hline
\end{tabular}

Table 2: Starting with well-founded estimation

Some of the pupils were able to stop experimenting in a qualified way exactly at the place where is stopped being meaningful (see Table 3):

\begin{tabular}{|c|c|c|c|c|}
\hline $\begin{array}{c}\text { Number of tickets } \\
\text { at 220 CZK }\end{array}$ & $\begin{array}{c}\text { Price in } \\
\text { CZK }\end{array}$ & $\begin{array}{c}\text { Number of tickets } \\
\text { at } 160 \text { CZK }\end{array}$ & $\begin{array}{c}\text { Price in } \\
\text { CZK }\end{array}$ & $\begin{array}{c}\text { Total price } \\
\text { in CZK }\end{array}$ \\
\hline 65 & 14300 & 32 & 5120 & 19420 \\
\hline 64 & 14080 & 33 & 5280 & 19360 \\
\hline $\mathbf{6 3}$ & $\mathbf{1 3 8 6 0}$ & $\mathbf{3 4}$ & $\mathbf{5 4 4 0}$ & $\mathbf{1 9 3 0 0}$ \\
\hline 62 & 13640 & 35 & 5600 & 19240 \\
\hline
\end{tabular}

Table 3: Qualified termination of experimenting

Answer: 63 tickets at the price of $220 \mathrm{CZK}$ and 34 tickets the price of $160 \mathrm{CZK}$ were sold in the theatre.

Let us now discuss two problems from category 3 above, i.e. problems where systematic experimentation results in verification of a hypothesis. 


\section{Problem 2}

Assignment: The numbers that are read the same from left to right and right to left, e.g. 452 254, are called palindromes. My friend claims that all four digit palindromes are divisible by 11 . Is it true?

Solution: Palindromes are e.g. numbers: 127 721, 94 749, 8 $338,565,44,8$. In the task, we are only interested in four digit numbers such as 6776,1 001, 2 992. Let us now take several four digit palindromes at random and divide them by eleven. We will get e.g. the following:

$$
\begin{aligned}
& 4554=414 \times 11 \\
& 1001=91 \times 11 \\
& 8338=758 \times 11
\end{aligned}
$$

It seems my friend could be right. However, we will not be one hundred percent sure unless we check all the 90 existing

\begin{tabular}{|c|c|c|c|c|c|}
\hline$a$ & $b$ & $"=b 2 " \omega$ & $"=a 2 "$ & $\begin{aligned} *= & 1000 * \mathrm{~A} 2+100 * \mathrm{~B} 2 \\
& +10 * \mathrm{C} 2+1 * \mathrm{D} 2 *\end{aligned}$ & $" '=E 2 / 11 "$ \\
\hline 1 & 0 & 0 & 1 & 1001 & 91 \\
\hline 1 & 1 & 1 & 1 & 1111 & 101 \\
\hline ... & $\cdots$ & $\cdots$ & $\cdots$ & $\ldots$ & $\cdots$ \\
\hline 2 & 9 & 9 & 2 & 2992 & 272 \\
\hline$\ldots$ & $\cdots$ & $\cdots$ & $\cdots$ & $\cdots$ & $\cdots$ \\
\hline 9 & 1 & 1 & 9 & 9119 & 829 \\
\hline$\cdots$ & $\cdots$ & $\cdots$ & $\cdots$ & $\cdots$ & $\cdots$ \\
\hline 9 & 9 & 9 & 9 & 9999 & 909 \\
\hline
\end{tabular}
four digit palindromes. This can be done efficiently using a spreadsheet (see Table 4).

Table 4: Use of spreadsheet for Problem 2

The last column shows that all quotients are whole numbers.

Answer: My friend was right.

In contrast, the use of spreadsheet can result in rejection of the hypothesis.

\section{Problem 3}

Assignment: Decide if the sequence $x_{n}=\frac{20^{n}}{n !}$ is monotonous. Solution: Let us determine the first few members of the sequence $x_{n}$.

$$
\begin{aligned}
& x_{1}=20 \\
& x_{2}=200 \\
& x_{3}=1333.3 \ldots \\
& x_{4}=6666.6 \ldots
\end{aligned}
$$

The natural hypothesis is that the sequence $x_{n}$ is increasing. Most pupils will come to this conclusion without hesitation.

Now it is the teacher's turn to remind pupils of the definition of an increasing sequence and to make pupils consider whether the conclusion they came to really corresponds to the condition in the definition that inequality $x_{n}<x_{n+1}$ holds for all natural numbers $n$. Only very few pupils will be able to realize at this point that it is not the case. However, using a spreadsheet they will see it all at once (see Table 5).

\begin{tabular}{|c|c|}
\hline$n$ & $x_{n}$ \\
\hline 1 & 20.0 \\
\hline 2 & 200.0 \\
\hline 3 & 1333.3 \\
\hline$\ldots$ & $\ldots$ \\
\hline 17 & 36850332.5 \\
\hline 18 & 40944813.9 \\
\hline 19 & 43099804.1 \\
\hline 20 & 43099804.1 \\
\hline 21 & 41047432.5 \\
\hline 22 & 37315847.7 \\
\hline 23 & 32448563.2 \\
\hline$\cdots$ & $\ldots$ \\
\hline
\end{tabular}

Table 5: Use of spreadsheet for Problem 3

Answer: It is obvious that the sequence $x_{n}$ is not monotonous. The member $x_{19}=x_{20}$ and the following members are decreasing.

\section{Guess - check - revise}

This strategy, similarly to the above discussed strategy, is from the family of experimental strategies. Its principle is very simple: we guess a solution, check it and make a new guess on the basis of the previous result. E.g. if a pupil is carrying out "manual" division of a multi digit number by a two digit number, he/she is using this strategy. At first he/she guesses how many times the divisor is present in the dividend and then checks this guess by calculation. If the estimation was not correct, he/she makes a new guess taking into account the previous unsuccessful guess and carries on in the same way. This practice of an important heuristic strategy is the reason why manual division should remain part of school mathematics even at the time of calculators and computers.

In case of arithmetic problems it is very convenient to record the results in a table. If there is a pattern or regularity pointing at a result it is more likely to be discernible in a table. The idea of the use of this strategy in arithmetic problems is pretty obvious. That is why we will illustrate this strategy on "graphic" solving procedures that are less common but may be much more efficient. Let us now illustrate the described strategy by two problems.

\section{Problem 4}

Assignment: There are two balls on a billiard table. Determine the place on the edge of the billiard table in such a way that the ball $A$ caroms ball $B$ in one rebound.

Solution: Experimenting is conducted using the GeoGebra. Figure 1 illustrates the situation from the assignment. The illustration may be created by the solver on his/her own if they know the principles of reflection or may be prepared by the author of the problem. Then the illustration may be an applet on a website and can be opened on the solver's device and experimented with.

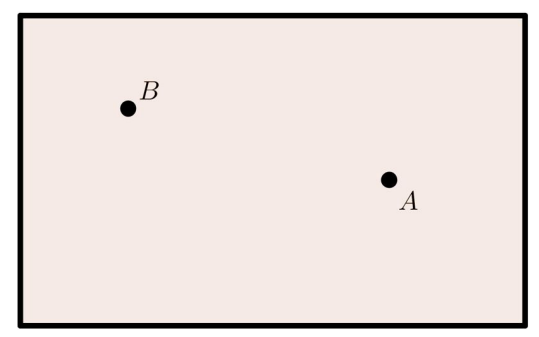

Figure 1: Situation from the assignment 
If we move the point on the edge of the table we will gradually arrive at the right solution (see Fig. 2). It follows a number of guesses only one of which is right (with respect to one edge).

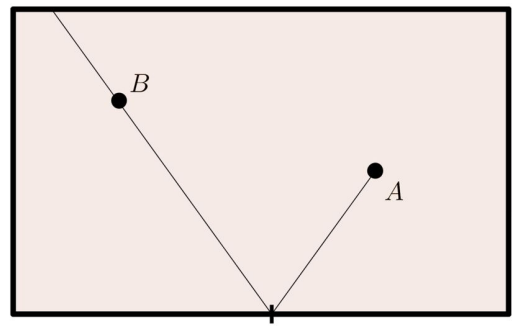

Figure 2: Moving the point on the edge of the table

Animated movement representing the rolling ball (represented by smaller point - see Fig. 3) is also possible.

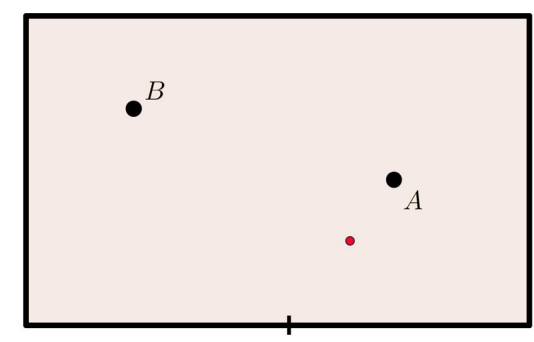

Figure 3: Animated movement

As soon as the pupil experimentally determines the right place on the edge of the table (let us call it point $C$ - see Fig. 4), he/ she can start thinking about the situation. The existence of four possible solutions - one for each edge - gives him/her insight into the situation from more points of view. This will be most illustrative if points $A, C$ and $B, C$ are connected by straight lines $p$ and $q$.

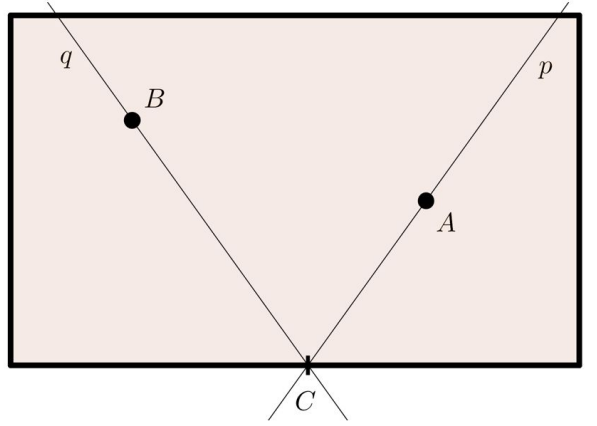

Figure 4: Finding the point $C$

We know that any two intersecting lines have two lines of symmetry (see Fig. 5).

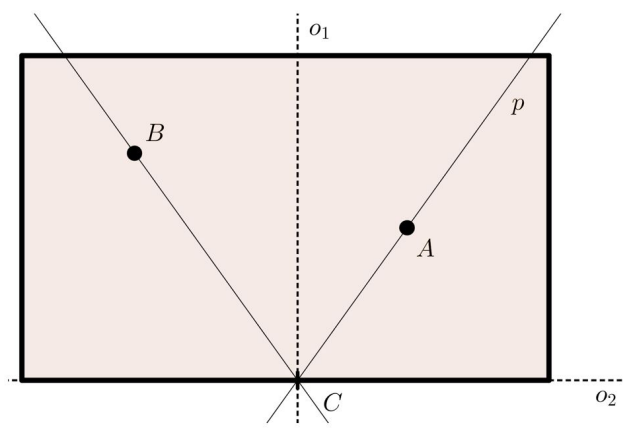

Figure 5: Lines of symmetry

The vertical line of symmetry $o$, represents the principle of reflection, the horizontal line $o_{2}$ allows us to solve the task. What we have to do is find the reflection of point $B$ in line symmetry $\mathrm{O}_{2}$. The connecting line of points $B^{\prime}$ and $A$ intersects the edge of the table in the sought point $C$ (see Fig. 6).

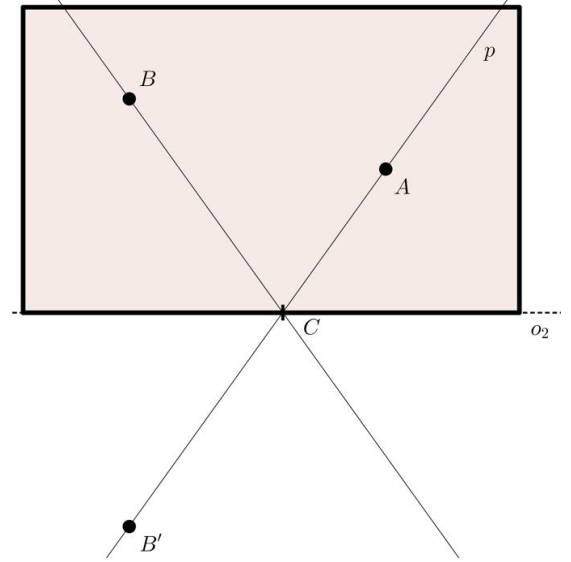

Figure 6: Finding the point $B$,

Experimenting helped us not only find the sought point but also the idea how to construct the sought point.

Answer: Point $C$ obtained as described above is the place we were looking for.

\section{Problem 5}

Assignment: An employee's monthly salary was 15755 CZK. During the year he got a pay raise of $2100 \mathrm{CZK}$. Since which month was his income higher if his yearly income was 195360 CZK?

Solution: Let us solve the problem by experimenting with the use of spreadsheet (see Tab. 6). Let us make a first guess and give the employee the pay raise in June (the third row in the table). The annual income is then higher than in the assignment. Let us try to give him the pay raise later - in November (the fourth row in the table). That is too little. So now, we can try to give him the pay raise in the tenth month (the fifth row in Table $6)$. Thus we got the requested income.

\begin{tabular}{|c|c|c|c|}
\hline & $\begin{array}{c}\text { Original } \\
\text { income }\end{array}$ & $\begin{array}{c}\text { Income after } \\
\text { pay raise }\end{array}$ & Annual income \\
\hline & 15755 & 17855 & 195360 \\
\hline Number of months & 5 & 7 & 203760 \\
\hline & 10 & 2 & 193260 \\
\hline & 9 & 3 & $\mathbf{1 9 5 3 6 0}$ \\
\hline
\end{tabular}

Table 6: Use of spreadsheet for Problem 5

Answer: The employee got the pay raise in October.

\section{Working backwards}

This is a very common strategy in mathematics. We assume that what we have to find/prove/construct holds/exists. Then we try to deduce from this assumption something we already know or something that is easy to prove/calculate/construct. Thus we in fact try to get from the end to the starting situation as close as possible. The procedure is reverted in the final calculation/ proof/construction. Let us now illustrate the described strategy by two problems.

\section{Problem 6}

Assignment: There are 16 matches in pile. Two players take turns to take one or two matches from the pile. The winner is the player who takes the last one or two matches. Find the winning strategy for the first player. (A winning strategy is a strategy which, if used by the player, results in victory of the player regardless of the moves of the opponent).

Solution: Let us proceed from the end of the game: If one of the players (let us call him/her $A$ ) manages to arrange that his/ 
her opponent (let us call him/her $B$ ) must play when there are 3 matches in the pile, $A$ will be the winner. Three matches in the pile are the closer target. Let us continue. If player $A$ manages to arrange that it is player $B$ 's turn when there are 6 matches in the pile, he/she will be the winner. Thus six matches in the pile after player $A$ 's turn is the new closer target. And analogically: even closer targets of the winning strategy are $9,12,15$. So if the first player plays in a way that after his/her move that are the following numbers of matches in the pile $-15,12,9,6,3$, he/she will be the winner.

Answer: If the first player first takes way one match and then plays in such a way that there are 15, 12, 9, 6, 3 matches after his/her move, he/she will be the winner.

\section{Problem 7}

Assignment: I am thinking of a number. If I add 300 and subtract 165 , I get a number which is five times greater than number 79 . Which number am I thinking of?

Solution: Let us first calculate the number we are meant to get to following the instructions:

$$
5 \times 79=375 .
$$

Now we will carry out inverse operations to the operations from the assignment:

$$
395+165-300=260 .
$$

Answer: The number is 260.

\section{Introduction of an auxiliary element}

The basic idea of this strategy is that the introduction of the so called auxiliary element makes the solution more easily accessible to the solver. In accordance with (Polya, 2004) we define an auxiliary element as an object that is not explicitly present in the problem assignment and that we introduce into the problem, hoping it will help us see the solution more easily. Polya calls this strategy Auxiliary elements and understands it in a broader context than as we present it because we focus exclusively on school problems. Introduction of an auxiliary element usually means adding lines, line segments, circular arcs or other geometrical figures. In case of algebraic problems we usually add a convenient number to both sides of the equation or we introduce a new unknown. As this element is not explicitly present in the problem assignment and we introduce it into it, we call this type of solution the strategy of Introduction of an auxiliary element. This strategy is very common in school mathematics and we are often not aware we are actually using it. Let us now illustrate the described strategy by two problems.

\section{Problem 8}

Assignment: Let $p$ be a prime number greater than 3. Prove that number $p^{2}-1$ is always divisible by the number 24 .

Solution: Let us first decompose the given quadratic binomial

$$
p^{2}-1=(p-1)(p+1) \text {. }
$$

Then let us insert number between numbers and ( $p$ is our auxiliary element). Let us now consider the triplet of numbers

$$
p-1, p, p+1
$$

They are three consecutive natural numbers. Thus one of them must be divisible by 3 . The prime number cannot be divisible by three. Therefore it is either or, which is divisible by 3 . Since numbers and are both even (the only even prime number is 2 ), they are both divisible by 2 and one must even be divisible by 4 . Therefore the product

$$
(p-1)(p+1)
$$

must be divisible by the product of number 3,2 and 4 , i.e. the number 24.

\section{Problem 9}

Assignment: Given is a convex quadrilateral $A B C D$, see Fig. 7. Join midpoints $M, N$ of the sides $A D$ and $B C$. Determine the relation between $|M N|$ and $\frac{1}{2}(|A B|+|C D|)$.

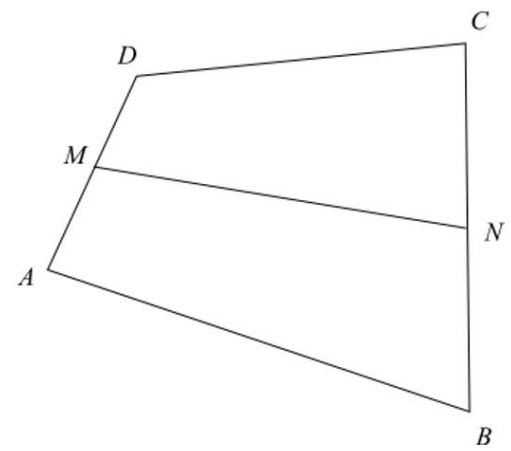

Figure 7: Quadrilateral $A B C D$

Solution: Let us introduce an auxiliary element into the assignment. Let this element be the diagonal $B D$ (see Fig. 8).

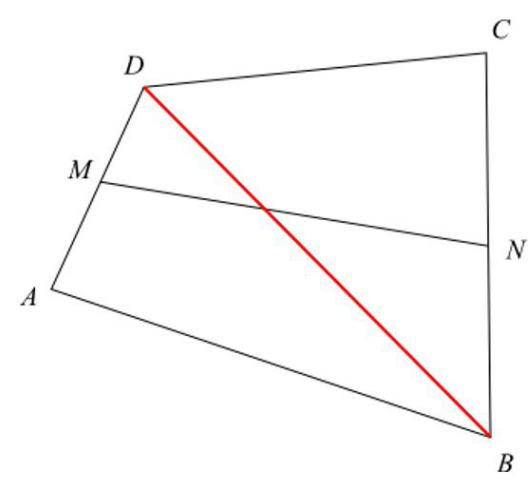

Figure 8: Used auxiliary element

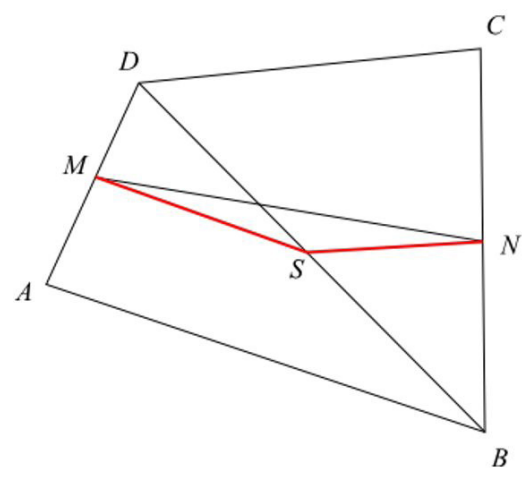

Figure 9: Triangles $A B D$ and $C D B$

Let us now focus on the situation in the created triangles. If we construct the midpoint $S$ on the diagonal $B D$, then the line segments $M S$ and $N S$ are the midlines of the triangles $A B D$ and $C D B$ respectively (see Fig. 9). Thus it holds that

$$
\begin{aligned}
& |M S|=\frac{1}{2}|A B|, \\
& |N S|=\frac{1}{2}|C D| .
\end{aligned}
$$


Applying triangle inequality to the triangle $S N M$ we get:

$$
|M N|<|M S|+|N S|=\frac{1}{2}|A B|+\frac{1}{2}|C D|=\frac{1}{2}(|A B|+|C D|) .
$$

Note: It is obvious that the relationship of equality comes when the point $S$ (the midpoint of diagonal $B D$ ) is on the line segment $M N$, i.e. in case that $A B C D$ is a trapezium.

Answer: It holds in any convex quadrangular $A B C D$, whose midpoints of the sides $A D$ and $B C$ are $M$ and $N$ respectively:

$$
|M N| \leq \frac{1}{2}(|A B|+|C D|) .
$$

\section{Omitting a condition}

Problem assignment often involves several conditions. If we are not able to fulfill all these conditions when solving the problem at once, we can ask similarly to Zeitz (2007): What is it that makes the solution of this problem so difficult? If we manage to identify which of the initial conditions is the difficult one, we can try to omit it. If we are then able to solve the simplified problem, we can go back to the omitted condition and try to finish solution of the original problem. Let us now illustrate the described strategy by two problems.

\section{Problem 10}

Assignment: Construct a rectangle $A B C D$, where the ratio of the sides is $3: 2$ and whose diagonal is $7 \mathrm{~cm}$ long.

Solution: If we omit the condition that "its diagonal is $7 \mathrm{~cm}$ long", we get a very simple problem. We construct the rectangle AKLM, whose sides are e.g. $3 \mathrm{~cm}$ and $2 \mathrm{~cm}$ (see Fig. 10). The assigned rectangle is an enlargement of the constructed rectangle with the centre $A$ (see Fig. 11). The vertex $C$ must lie on the ray $A L$ in the distance of $7 \mathrm{~cm}$ from the vertex $A$. The following construction is obvious.

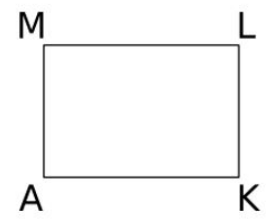

Figure 10: Rectangle $A K L M$

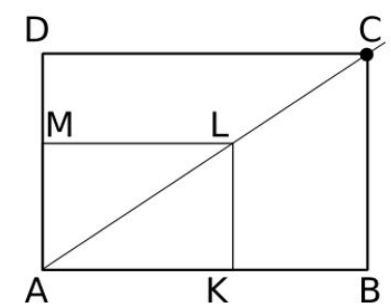

Figure 11: Enlargement with the centre $A$

\section{Problem 11}

Assignment: Given are a classical chessboard from which two opposite corner black fields have been cut off (see Fig. 12) and a sufficient number of domino tiles. Is it possible to cover this cut off chessboard with domino tiles in such a way that none of them sticks out of the board?

Solution: Let us omit the condition that the two fields have been cut off and let us work with a classical chessboard of 64 fields. It is relatively easy to cover this chessboard with domino tiles. Let us now return to the omitted condition - let us cut off the two opposite corner black fields from the (covered) chessboard. Obviously together with the two fields also two domino tiles will be removed and two white fields will remain uncovered. As each tile can only cover one black and one white field, the two remaining white fields obviously cannot be covered with a domino tile however we place the other domino tiles.

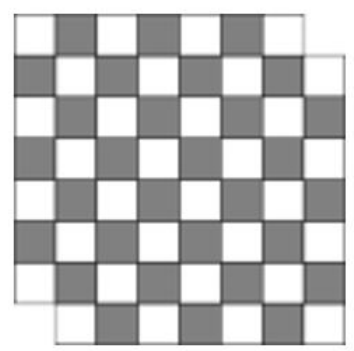

Figure 12: The given board

Answer: The cut off chessboard cannot be covered with domino tiles.

While strategies Introduction of an auxiliary element and Omitting a condition require creative activity from the solver and depend on the solved problem, the first three strategies can be characterized as strategies of algorithmic nature and pupils can use them successfully even if they do not have very good insight into the structure of the problem; use of these strategies does not always ask for very active involvement of pupils' creativity.

The participating teachers were provided with a sufficient number of problems that are solved most efficiently using one of the above listed strategies.

The teachers' work was organized as follows. They assigned a problem to their pupils. They let them work and asked the pupil who was the fastest to solve the problem correctly to explain their solution to the others. This was followed by a discussion and explanation of the solving strategy. The teacher then asked other successful solvers to present alternative solutions to the others. If none of the pupils solved the problem with the intended heuristic strategy, it was demonstrated by the teacher. In another, similar problem the teacher then checked to what extent the teacher's solution was actively understood.

Every teacher solved in this way about three problems a week. The pupils sat a written pre-test and post-test at the beginning and the end of the experiment. The problems in the test were the same. Each test consisted of four tasks which were about equally as difficult as problems solved during the teaching experiment. All the problems in the test shared one feature. The selected heuristic strategy was the most efficient way of solving the problem. When evaluating the written tests, attention was paid to success rate but also to the method of solution, i.e. also whether the pupils used some of the strategies shown in the teaching experiment.

\section{Results and Discussion}

\section{Research questions}

1. Is it possible to achieve any progress in the ability to solve mathematical problems using the strategies Introduction of an auxiliary element and Omitting a condition for a short period of time (4 months) or do they make their "implantation" in such a short period of time impossible?

2. The strategy that seemed as the most easily usable in the experiment was Systematic experimentation (see Novotná et al., 2014). Does its frequent use by pupils have only positive consequences, or does it bear any potential risks? If so, which? 


\section{Results}

Because of the limited scope of the paper, we have chosen one or two problems from a test in each age category which we present and comment upon.

\section{2-year-old pupils, 98 respondents in total:}

\section{Problem 1}

Assignment: Inscribe a square $K L M N$ in a given triangle $A B C$ (see Fig. 13). Two vertices $(K, L)$ of the square should be on the base $A B$ of the triangle, the two other vertices $(M, N)$ of the square on the two other sides of the triangle, one on each. (Polya, 2004, p. 23)

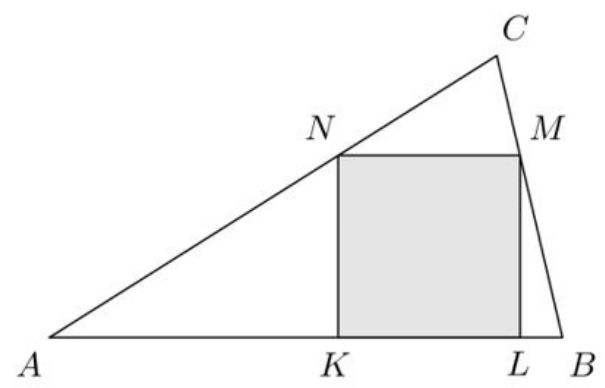

Figure 13: Assigned objects

Efficient solving strategy: Omitting a condition.

It is unlikely we succeed in constructing a square executing all conditions. If we drop the "the lies on the side" condition, we may find constructing such a $K_{1} L_{1} M_{1} N_{1}$ square with ease (see Fig. 14). We inscribe it in the $B A C$ angle. The square we search is to be homothetic to that, with homothetic centre in the $A$ point. The point $M$ is the point of intersection of the ray $A M_{1}$ with the $B C$ side.

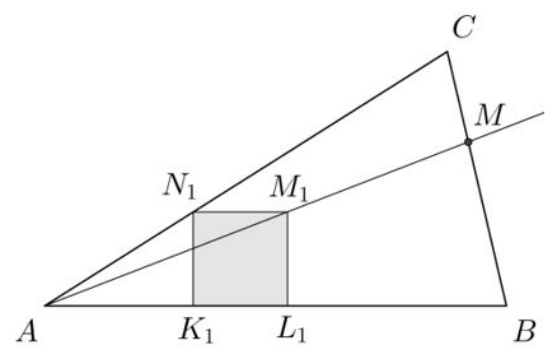

Figure 14: Constructing the square

Success rate in pre-test: $9 \%$.

Success rate in post-test: $20 \%$.

The number of pupils who used the strategy Omitting a condition in the pre-test: $9 \%$.

The number of pupils who used the strategy Omitting a condition in the post-test: $20 \%$.

\section{Problem 2}

Assignment: Determine the area of the kite whose measurements are given in Fig. 15. The data are given in centimeters.

Efficient solving strategy: Introduction of an auxiliary element.

We add diagonals into the kite figure (see Fig. 16) and use the formula for calculation of the area of a triangle. Let us use letters $a, b, c$ in this order to label the sizes of the corresponding diagonals.

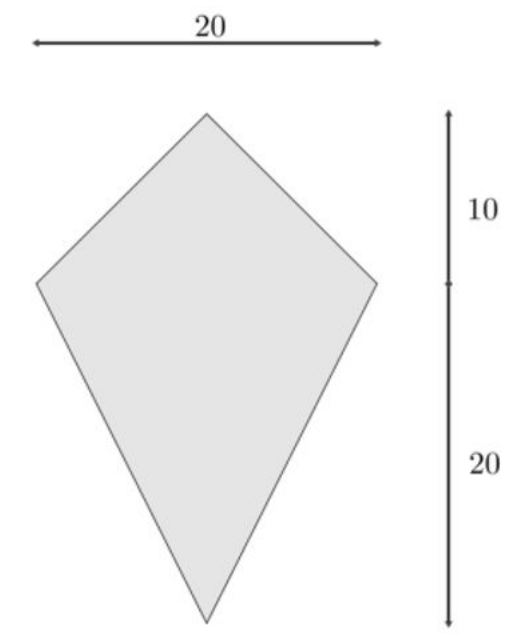

Figure 15: Figure assigned in Problem 2

$$
\begin{gathered}
S_{1}=\frac{a \times b}{2} \\
S_{2}=\frac{b \times c}{2} \\
S=2 S_{1}+2 S_{2}
\end{gathered}
$$

The answer is: We need $300 \mathrm{~cm}^{2}$ of paper to create the kite. Success rate in pre-test: $13 \%$.

Success rate in post-test: $58 \%$.

20

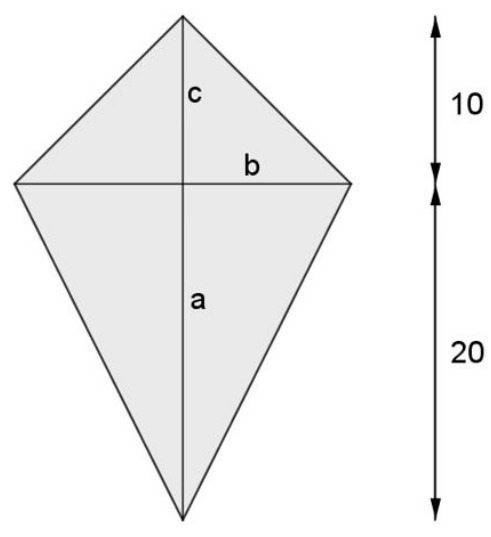

Figure 16: Auxiliary elements - diagonals

The number of pupils who used the strategy Introduction of an auxiliary element in the pre-test: $18 \%$.

The number of pupils who used the strategy Introduction of an auxiliary element in the post-test: $74 \%$.

\section{4-year-old pupils, 109 respondents in total:}

\section{Problem 3}

Assignment: Find two consecutive natural odd numbers whose product is 1023. (Cihláŕ, Zelenka, 1998, p. 89/12)

Efficient solving strategy: Systematic experimentation.

We select pairs of odd numbers in Table 7 and look for their product. 


\begin{tabular}{|c|c|c|}
\hline First odd number & Second odd number & Product of the numbers \\
\hline 1 & 3 & 3 \\
\hline 3 & 5 & 15 \\
\hline 5 & 7 & 35 \\
\hline 7 & 9 & 63 \\
\hline 9 & 11 & 99 \\
\hline 11 & 13 & 143 \\
\hline 13 & 15 & 195 \\
\hline 15 & 17 & 255 \\
\hline 17 & 19 & 323 \\
\hline 19 & 21 & 399 \\
\hline 21 & 23 & 483 \\
\hline 23 & 25 & 575 \\
\hline 25 & 27 & 675 \\
\hline 27 & 29 & 783 \\
\hline 29 & 31 & 899 \\
\hline 31 & $\mathbf{3 3}$ & $\mathbf{1 0 2 3}$ \\
\hline $1967:$ & $5 y$ & \\
\hline
\end{tabular}

Table 7: Problem - Systematic experimentation

The answer is: The sought numbers are 31 and 33.

Success rate in pre-test: $21 \%$.

Success rate in post-test: $54 \%$.

The number of pupils who used the strategy Systematic experimentation in the pre-test: $0 \%$.

The number of pupils who used the strategy Systematic experimentation in the post-test: $27 \%$.

\section{7-year-old pupils, 78 respondents in total:}

\section{Problem 4}

Assignment: The perimeter of a rectangular garden is $114 \mathrm{~m}$. One of its sides is $13 \mathrm{~m}$ longer than the other. Find the area of this garden.

Efficient solving strategy: Omitting a condition combined with Systematic experimentation.

Let us omit the explicitly stated condition that the perimeter of the garden is $114 \mathrm{~m}$. Now we will use the strategy Systematic experimentation. Let us create a table whose first column represent length of side $a$, second column length of side $b=a+13$, third column perimeter $P=2(a+b)$ and fourth column area $S=a b$ (see Table 8 ). The table can be easily made e.g. in Excel, where after setting the formulae the program constructs the table within seconds.

\begin{tabular}{|c|c|c|c|}
\hline$a$ & $b$ & $P$ & $S$ \\
\hline 1 & 14 & 30 & 14 \\
\hline 2 & 15 & 34 & 30 \\
\hline$\ldots$ & $\ldots$ & $\ldots$ & $\ldots$ \\
\hline 21 & 34 & 110 & 714 \\
\hline $\mathbf{2 2}$ & $\mathbf{3 5}$ & $\mathbf{1 1 4}$ & $\mathbf{7 7 0}$ \\
\hline 23 & 36 & 118 & 828 \\
\hline$\ldots$ & $\ldots$ & $\ldots$ & $\ldots$ \\
\hline 44 & 57 & 202 & 2508 \\
\hline
\end{tabular}

Table 8: Problem 4-Omitting a condition combined with Systematic experimentation

If we reconsider the omitted condition we easily find the solution (it is highlighted in the table).

The answer is: The area of the garden is $770 \mathrm{~m}^{2}$.

Success rate in pre-test: $27 \%$.

Success rate in post-test: $39 \%$.

The number of pupils who used the strategies Systematic experimentation and Omitting a condition in the pre-test: $0 \%$.

The number of pupils who used the strategy Systematic experimentation and Omitting a condition in the post-test: $15 \%$.

In the pre-test, successful students solved it exclusively using equations. However, although the problem is quite simple, many students failed to construct the equations correctly. In the post-test the number of successful solvers increased by students who combined strategies Omitting a condition and Systematic experimentation. Most of the pupils using combination of these strategies were successful.

\section{Conclusion}

The results of the experiment gained from the pre-tests and posttests as well as from interviews with participating teachers allow us to formulate the following conclusions:

Experimental strategies (Guess - check - revise, Systematic experimentation) and the strategy Introduction of an auxiliary element can be mastered already in shorter period of time, the strategy Omitting a condition requires longer time. This is caused by algorithmic nature of the two experimental strategies (Novotná et al., 2014). As far as the strategy Introduction of an auxiliary element is concerned, the pupils were quite fast to learn to add different auxiliary elements into the drawing, not to give up solving the problem, to experiment.

The danger of Systematic experimentation is that its mastery by some pupils makes them use it as first solving procedure instead of e.g. constructing equation or system of equations. On the other hand, more frequent use of the strategy Systematic experimentation develops pupils' sense of effective choice of the initial value.

The short period of time of the experiment was sufficient to change attitudes of some pupils and students to problem solving (this could usually be observed in about one half of the pupils and students in each class). Pupils and students stopped being afraid of solving problems, they stopped laying their solution aside if they were not sure how to solve them in the very beginning. They learned to look for a solution rather than to give up.

Some teachers (about one third) claim that thanks to this experiment the ability to express ideas improved in about one half of their pupils or students. This is confirmed by the fact that pupils and students tend to comment on their solutions in more detail in the post-tests, more of them try to justify the individual steps in their solving procedure.

\section{Acknowledgements}

The research was supported by the project GAČR P407/12/1939.

The described teaching experiment was conducted in classes of these teachers: Miroslav Mázl, Roman Marschner, Vojtěch Oliverius, Pavla Linková, Iva Hotová, Hana Amlerová and Jiř́ Bureš. The authors would like to thank all these teachers and their pupils for their conscientious attitude to solution of the research problem. 


\section{References}

Brousseau, G., Novotná, J. (2008) 'La culture scolaire des problèmes de mathématiques', Les didactiques et leurs rapports à l' enseignement et à la formation. Quel statut épistémologique de leurs modèles et de leurs résultats?, Bordeaux, CD ROM.

Břehovský, J., Eisenmann, P., Ondrušová, J., Přribyl, J. and Novotná, J. (2013) 'Heuristic Strategies in Problem Solving of 11-12-year-old pupils', Proceedings of SEMT '13, Prague, pp. 75-82.

Cihlář, J., Zelenka, M. (1998) Matematika 8, Praha: Pythagoras Publishing, a.s.

Elia, I., van den Heuvel-Panhuizen, M. and Kolovou, A. (2009) Exploring strategy use and strategy flexibility in nonroutine problem solving by primary school high achievers in mathematics. ZDM Mathematics Education, 41(5), pp. 605-618. Fan, L.and Zhu,Y. (2007) Representation of Problem-Solving Procedures: A Comparative Look at China, Singapore, and US mathematics textbooks. Educational Studies in Mathematics, 66(1), pp. 61-75.

Novotná, J., Eisenmann, P., Přibyl, J., Ondrušová, J. and Břehovský, J. (2013) 'Heuristic strategies in problem solving in school mathematics', Proceedings of the 10th International Conference on Efficiency and Responsibility in Education (ERIE 2013), Prague, pp. 461-468.

Novotná, J., Eisenmann, P., Přibyl, J., Ondrušová, J. and Břehovský, J. (2014) 'Problem solving in school mathematics based on heuristic strategies', Journal on Efficiency and Responsibility in Education and Science, vol. 7, no. 1, pp.1-6.

Pólya, G. (2004) How to Solve It: A New Aspect of Mathematical Method (Expanded Princeton Science Library ed.). Princeton: Princeton University Press.

Wittmann, E.Ch. (1995) 'Mathematics education as a „Design Science", Educational Studies in Mathematics, vol. 29, no. 4, pp. 355-374.

Zeitz, P. (2007) The Art and Craft of Problem Solving, New York: John Wiley \& Sons, Inc. 